Annals

Spor

www.aassjournal.com

ISSN (Online): 2322 - 4479

ISSN (Print): 2476-4981
Ariginal Article

www.AESAsport.com

Received: 28/04/2017

Accepted: 19/07/2017

\title{
The Variation of Acquisition, Consolidation Memory and Coordination Pattern of Elbow Joint in Short Service Badminton before and after Aerobic Training
}

\author{
${ }^{1}$ Teimour Darzabi, ${ }^{1}$ Hamid Reza Taheri ${ }^{*}$, ${ }^{1}$ Ali Reza Saberi Kakhki \\ ${ }^{1}$ Department of Motor Behavior, Faculty of Sport Sciences. Ferdowsi University Of Mashhad, Mashhad, Iran.
}

\begin{abstract}
Background. Consider the fact that the implementation of aerobic exercise can be effective in the learning and acquisition process, and that training athletes to acquire new skills is appropriately done in terms of the primacy and recency of the training time for aerobic activities such as the warm-up. Objectives. The purpose of the present study was to investigate the effect of moderate-intensity aerobic activity before training on the acquisition, consolidation memory, and coordination pattern of the elbow joint with regard to the short service in badminton. Methods. Thirty-eight participants aged 17 to 19 years were selected by convenience sampling and purposive sampling. The participants were randomly assigned to two experimental groups $(n=14$ and $n=10)$ and a control group $(n=14)$ based on aerobic capacity. Before and after the badminton short service training, the participants ran for 20 minutes on a treadmill at a speed of about $70 \%$ of their maximum heart rate. At various stages of training, the precision of the shuttlecock landing spots was measured based on the French test at the time of acquisition, immediate retention, retention, and transfer in all three groups. Also, using motion analysis, the range of motion of the wrist, elbow, and shoulder joints was measured. Moreover, by using a PCA, the coordination pattern of the range of motion of the elbow joint was compared in all three groups. Results. There was no significant difference between the groups in terms of the accuracy of the short service in badminton in the French test based on aerobic exercise before and after training ( $p<0.05)$.A significant difference was observed between all parts of the test and the transfer test $(\mathrm{p}>0.05)$. Also, there was no significant difference in the coordination pattern of the range of the elbow joints between the groups. Furthermore, the repeat of service has no significant effect on the motion pattern in any way. Conclusion. According to the impact of moderate-intensity aerobic activity before and after training on the acquisition, consolidation of memory, and coordination pattern of the elbow joint, there was no significant difference in the accuracy of the short service in badminton.
\end{abstract}

KEY WORDS: Aerobic Activity, Acquisition, Consolidation Memory, Short Service Badminton.

\section{INTRODUCTION}

Motor learning is a set of processes associated with the practices and experiences leading to relatively constant changes in the ability to carry out movements. A process is a series of events that leads to certain results, states, or changes (1).
One of the main objectives of the study of cognitive learning is to identify dependent variables that may enhance or disrupt the learning, or that may play no role in it. It is necessary to identify these variables related to

*. Corresponding Author:

Hamid Reza Taheri

E-mail: hamidtaheri@ferdowsi.um.ac.ir 
learning and practical applications in training and other learning opportunities (1).

In this regard, the researchers of motor learning sought to examine the factors that enhance the performance of motor skills over time. One factor that affects the formation of memory and motor learning is practicing skills with physical activity (2-4). Participating in physical activities such as aerobic exercises can particularly affect the formation of memory and motor learning (5). In this context, participation in regular physical activity may be considered one of the best solutions available for learning and memory (6). The results show that the potential impact of intense exercise on identifying and encoding procedural information during exercise after the training is not transmitted immediately and is not observed at the time of acquisition. Hence, it can be useful to implement a period of intense exercise related to motor tasks that focus on accuracy and the acquisition of motor skills (3). In fact, recent evidence suggests that moderate-intensity exercise has harmful effects on accuracy (3). Therefore, the high arousal level enhances the process of encoding that temporarily inhibits data recovery through memory consolidation (7). This process has destructive effects on short-term memory (8). Another possibility is that the memory representation of motion is still not stabilized when the first retention test is run. Therefore, the positive impact of intense exercise on memory is not yet fully proven, and there is a contradiction between immediate and delayed retention tests, which is not unusual in studies of learning motor skills (9).

Little research has focused on the position of aerobic exercise in training programs. Some research has examined the effect of aerobic exercise intervention on the short serve in badminton using the cognitive task $(3,10)$ or the tracking task (11) and the continuous task. These studies have shown different results on the effect of aerobic exercise on motor tasks. In this context, Labban et al. (2011) reported that performing aerobic exercises before training can be more effective than exercise after training (12). In contrast, Roig et al. (2012) examined the effect of moderate-intensity aerobic exercises on elbow torque. They concluded that there is no short-term effect of aerobic exercise and improved performance can be seen only in the delayed retention. They used the tracking task for visual-motor accuracy. Thus, a lever was used to show torque and angle changes, and track the target on the screen from left to right. The findings of this study showed no significant effect but they indicated improvement in the accuracy of the visual-motor task over time. Other studies have shown that aerobic exercise improves delayed retention in motor skills (11). These findings were approved for adolescents (9.5 years), middle-aged people (19-25 years), and elderly people (60-74 years) $(13,14)$. A study investigated the impact of aerobic activities on cognitive performance through the comparison of two methods: cycling and running on a treadmill. The findings indicated that cycling-based aerobic exercises enhance performance during and after exercise but treadmill-based aerobic exercises cause disturbance during exercise and result in little improvement in performance after exercise. The results indicated a complex relationship between exercise and cognitive performance, and increased performance depends on the type of cognitive skills and the type of exercise (15).

Among the subjects in the mentioned studies were biomechanical variables but the pattern of coordinated movement has not been studied. In other words, coordination and variability also seem to be important in explaining the results. The adjustment of coordinated movement is necessary for dynamic performance (16). The relationship between coordination variability and task has been examined in non-related badminton studies $(17,18)$. It is also suggested that coordination variability indicates the flexibility of the system to seek optimal performance $(17,18)$. Consider the fact that the implementation of aerobic exercise can be effective in the learning and acquisition process, and that training athletes to acquire new skills is appropriately done in terms of the primacy and recency of the training time for aerobic activities such as the warm-up. Some trainers perform the training at the beginning of the training session while others do it after the warm-up and aerobic activities, and even at the end of the training session. Therefore, a question arises: What impact can moderateintensity aerobic activity, such as a warm-up before or after a skills training session, have on the learning process? 


\section{MATERIALS AND METHODS}

Participants. The study was an applied research based on the quasi-experimental design. Thus, two experimental groups and one control group were compared. The sample included 38 male students aged between 17and 19years who lived in the city of Mashhad. In the first step, the participants were acquainted with the nature and the process of the study. The inclusion criteria were as follows: being healthy based on health questionnaire, no smoking, and lack of familiarity with badminton. The participants voluntarily participated in the study and signed a consent form. They were randomly assigned to two experimental groups $(\mathrm{n}=14$ and $\mathrm{n}=10)$ and a control group $(n=14)$ based on the results of the Bruce and Raven IQ test. Their body mass index was in the range of $21.30 \pm 2.55$; their intelligence was in the range of $115.24 \pm 9.67$; and maximal oxygen uptake was in the range of $44.90 \pm 7.05$ $\mathrm{mL} / \mathrm{kg}$ per minute.

Instruments and Equipment. The height of the participants was measured by the digital stadiometer seca (made in Germany) with a sensitivity of $5 \mathrm{~mm}$ to calculate the body mass index (BMI).The participants did not eat or drink for four hours before the test, and possibly their bladder, stomach, and intestines were discharged. The participants' IQ was measured by the Raven IQ test. The Raven IQ test included 60 image questions (A12, B12, C12, D12, E12) and each question had six to eight options. The scores for the60 questions were calculated to measure the participants' IQ. The correlation coefficient of the test was measured by the Stanford and Wechsler tests $(0.4-0.75)$, and the validity was $0.7-0.9$ for the older participants (19).

Maximal Oxygen Uptake. The maximal oxygen uptake was one of the inclusion criteria. It was measured by the Bruce test. In this 10-step method, the treadmill was started at $2.74 \mathrm{~km} / \mathrm{hr}$ (1.7 mph) and at a gradient (or incline) of $10 \%$. The speed and incline progressively increased until the participant was exhausted and unable to run. The maximal oxygen uptake in the Bruce test was calculated using Equation 1.

Equation 1: Maximal oxygen uptake $(\mathrm{ml} / \mathrm{kg} / \mathrm{min})$ $=14.8-(1.379 \times \mathrm{T})+(0.451 \times \mathrm{T} 2)-(0.012 \times \mathrm{T} 3)$
French Badminton Test. The French badminton test was used to measure the score forthe short serve (20). This test evaluates the accuracy of the short serve in badminton with respect to the ball landing in the confluence of the length line and the short service line.The Qualisys motion analysis system made by Qualisys AB, 2013-12-12, was used to measure the actual landing distance from the point and a phosphorus marker with awidth of $5 \mathrm{~mm}$ was installed on the perimeter of the shuttle ball. Yonex rackets and balls approved by Ball Badminton Federation, and a net with a height of 1.55 meters and a length of 6.10 meters were used for the short serve in badminton.

Procedure. Each of the groups participated in an initial training session, including on how to hold a racket correctly, and familiarity with the court, ball, and rally. Each group was scheduled for the following stages: training, acquisition, immediate retention, retention, and transfer. All activities of the groups were done in the morning to ensure that there was no difference in runtime. In the first session, the participants were trained to perform the Beck India Short Service in badminton. The four blocks of 10 trials were conducted by specifying the ball landing area based on the French test in badminton. There was a five-second rest between each trial and a five-minute rest between each block (20). The participants were given the same description and feedback during each step. The control group did not take any action before and after the training and the trials. Experimental Group $1(n=14)$ before training and Experimental Group $2(\mathrm{n}=10)$ after 40 trials ran on a treadmill for 20 minutes. The first and last three minutes were under lower pressure for the warm-up and warm-down. They ran for 14 minutes at $65 \%$ of maximal oxygen consumption. The second session (continuation of the process of acquisition and acquisition test) was held for each group after 48 hours. Again, the participants performed four blocks of 10 trials (without any aerobic activity).At the end of 40 trials in the second session, each participant rested for 40 minutes and then each participant performed 10 trials to review the immediate retention. The third session was held for each group after 72 hours. Again, the participants performed 10 trials. The fourth session was held for each group after 96 hours. Again, the participants performed 10 trials 
as before. They performed 10 trials by changing the ball landing area to check the transfer. Before performing the trials, all the groups performed the same stretching and flexibility exercises for five minutes. The data was collected based on lining the badminton court and the French test on the ball landing area. The scores were recorded using the Qualisys motion analysis system.

Information Processing. Information processing was done using MATLAB software. In order to smooth out the information, the fourthorder Butterworth low-pass filter with a cutoff frequency of $12 \mathrm{~Hz}$ was used. The range of movement for the shoulders, elbows, and wrists was measured (21). After measuring the range of movement for the joints, a principal component analysis was used to determine the variability of the pattern for coordinated movement (22). Principal component analysis is a statistical procedure that uses an orthogonal transformation to convert a set of variables into uncorrelated principal components. Each principal component explains some of the variances in the data; hence, the first principal components explain more variances. The contribution of each variable in the formation of each principal component is represented by coefficients. Finally, the elbow coefficients were extracted from the angular pattern of badminton skill in pre-test, acquisition, immediate reminder, and post-retention after five days, retention after nine days, and transfer.

Statistical Analysis. The collected data was analyzed using SPSS (version 16). After confirmation of the normal distribution of the data using the Kolmogorov-Smirnov (K-S) test and the homogeneity of variance using Levene's test, the repeated measures ANOVA was used to compare the between-groups mean and the within-groups mean. The results were analyzed at a significance level of $p<0.05$.

\section{RESULTS}

The characteristics of the subjects are shown in Table 1. There was no significant differences between the three groups before the intervention in terms of age, height, weight, IQ test, and maximal oxygen consumption $(\mathrm{p}>0.05)$.

Table 2 shows the results of the ball landing area at the confluence of the long line and the short line in the badminton court in millimeters for the three groups. According to the French short serve test, the lower the distance of the ball landing to the point, the higher the accuracy.

Table 1. Participant characteristics at baseline (Mean \pm SD)

\begin{tabular}{cccccc}
\hline Groups & $\begin{array}{c}\text { Age } \\
(\text { years })\end{array}$ & $\begin{array}{c}\text { Height } \\
(\mathrm{m})\end{array}$ & $\begin{array}{c}\text { Weight } \\
(\mathrm{kg})\end{array}$ & Body Mass Index (kg/m2) & Raven IQ Test \\
\hline Experimental 1 & $17.67 \pm 0.52$ & $175.30 \pm 3.56$ & $63.50 \pm 7.55$ & $44.36 \pm 6.82$ & $112.93 \pm 11.06$ \\
Experimental 2 & $17.80 \pm 0.45$ & $173.83 \pm 5.84$ & $66.58 \pm 14.13$ & $44.90 \pm 7.85$ & $115.20 \pm 8.31$ \\
Control & $17.5 \pm 0.58$ & $174.00 \pm 6.78$ & $66.31 \pm 10.63$ & $43.57 \pm 7.67$ & $119.46 \pm 5.87$ \\
\hline
\end{tabular}

Table 2. Measurement accuracy of short service badminton in the five stage test in three groups (Mean \pm SD)

\begin{tabular}{|c|c|c|c|c|c|}
\hline Groups & $\begin{array}{c}\text { The accuracy of } \\
\text { short service } \\
\text { badminton } \\
\text { (Pretest) }\end{array}$ & $\begin{array}{c}\text { The accuracy of } \\
\text { short service } \\
\text { badminton } \\
\text { (Acquisition } \\
\text { phase) }\end{array}$ & $\begin{array}{c}\text { The accuracy of } \\
\text { short service } \\
\text { badminton } \\
\text { (Immediate } \\
\text { retention phase) }\end{array}$ & $\begin{array}{l}\text { The accuracy of } \\
\text { short service } \\
\text { badminton } \\
\text { (Remember } \\
\text { phase after next } 5 \\
\text { days) }\end{array}$ & $\begin{array}{l}\text { The accuracy of } \\
\text { short service } \\
\text { badminton } \\
\text { (Remember phase } \\
\text { after next } 9 \text { days) }\end{array}$ \\
\hline Exp 1 & & & & $720.701 \pm 226.67$ & \\
\hline $\operatorname{Exp} 2$ & & $676.024 \pm 170.79$ & $722.365 \pm 124.49$ & 690. & $700.950 \pm 107.53$ \\
\hline Control & $752.387 \pm 187.49$ & $622.527 \pm 147.21$ & $662.089 \pm 182.98$ & $656.672 \pm 154.07$ & $645.012 \pm 137.27$ \\
\hline
\end{tabular}

Exp 1: Activities before education. Exp 2: Activities after education. The ball landing area to the confluence of the long line and short line in badminton court in millimeters

According to Table 3, there is no significant difference in the effect of aerobic activity before and after the short serve training at all stages of acquisition $(p=0.563)$ and the effect of memory 
consolidation on the accuracy of the short serve ( $p>0.05)$. The results show that repeating the serve has a significant effect on the accuracy of the short serve in badminton $(\mathrm{P}=0.001, \mathrm{~F}=4.920)$. According to the results of the least significant difference (LSD) test, there is a significant difference between the pre-test and other steps. There was no significant difference between the various methods (aerobic activity before and after training) $(\mathrm{P}=0.426, \mathrm{~F}=0.875)$. The number of repeats at different times had no significant effect on the different methods $(\mathrm{P}=0.097, \mathrm{~F}=0.999)$. The results of the elbow coefficients in the angular pattern of badminton are shown in Table 4. According to Table 5, the effect of aerobic activity before and after low serve badminton training was not significant on all stages of acquisition $(p=0.218)$ and memory consolidation between three groups for the elbow coefficients in the angular skill pattern $(\mathrm{p}<0.05)$.

\section{DISCUSSION}

This study aimed to investigate the impact of moderate-intensity aerobic activity before training on the acquisition and consolidation of memory for the short service in badminton. The results of the study indicated that aerobic exercise has no significant effect on the accuracy of the acquisition and retention of the short serve in badminton. The findings indicated that aerobic exercise after training can lead to a drop in the accuracy of the immediate retention. These results were consistent with the findings of the studies conducted by Roig et al. (2012) and Cian et al. (2001) $(8,11)$. Roig et al. (2012) reported that moderate-intensity aerobic exercise does not have a significant effect on the performance of the tracking task (11). These results were inconsistent with the findings of studies indicating that aerobic exercise has a positive effect on the acquisition of motor skill accuracy (23-25).

Table 3. Comparison of within group variance and between group of accuracy of short service badminton in separate groups during test (Mean \pm SD)

\begin{tabular}{lcccccc}
\hline \multicolumn{1}{c}{ Stages } & Variations & Sum squares & df & Mean Square & F & P-value \\
\hline Pretest & 1 & 48661.156 & 2 & 24330.578 & \multirow{2}{*}{0.488} & \multirow{2}{*}{0.618} \\
& 2 & 1746301.852 & 35 & 49894.339 & & \\
Acquisition phase & 1 & 38484.974 & 2 & 19242.487 & 0.584 & 0.563 \\
& 2 & 1152535.291 & 35 & 32929.580 & & \\
Immediate retention phase & 1 & 24888.469 & 2 & 12444.235 & 0.466 & 0.631 \\
Remember phase after next 5 & 2 & 934299.481 & 35 & 26694.271 & & \\
days & 1 & 28720.873 & 2 & 14360.436 & 0.441 & 0.647 \\
Remember phase after next 9 & 2 & 1139204.678 & 35 & 32548.705 & & \\
days & 1 & 21471.542 & 2 & 10735.771 & \multirow{2}{*}{0.441} & 0.647 \\
\hline
\end{tabular}

$\ddagger$ The mean difference is significant at the 0.05 level. 1) Between group. 2) Within group.

Table 4. Comparison of within group variance and between group of PCA angle elbow in separate groups during test $(\mathbf{M} \pm \mathrm{SD})$

\begin{tabular}{cccccc}
\hline Groups & $\begin{array}{c}\text { The PCA angle } \\
\text { elbowof short } \\
\text { service } \\
\text { badminton } \\
\text { (Pretest) }\end{array}$ & $\begin{array}{c}\text { The PCA angle } \\
\text { elbowof short } \\
\text { service } \\
\text { badminton } \\
\text { (Acquisition } \\
\text { phase) }\end{array}$ & $\begin{array}{c}\text { The PCA angle } \\
\text { elbowof short } \\
\text { service } \\
\text { badminton } \\
\text { (Immediate } \\
\text { retention phase) }\end{array}$ & $\begin{array}{c}\text { The PCA angle } \\
\text { elbowof short } \\
\text { service } \\
\text { badminton } \\
\text { (Remember } \\
\text { phase after next } \\
\mathbf{5} \text { days) }\end{array}$ & $\begin{array}{c}\text { The PCA angle } \\
\text { elbowof short } \\
\text { service } \\
\text { badminton } \\
\text { (Remember } \\
\text { phase after next } \\
\text { 9 days) }\end{array}$ \\
\hline Exp 1 & $0.683 \pm 0.240$ & $0.654 \pm 0.233$ & $0.625 \pm 0.249$ & $0.620 \pm 0.285$ & $0.616 \pm 0.295$ \\
Exp 2 & $0.596 \pm 0.307$ & $0.530 \pm 0.294$ & $0.651 \pm 0.287$ & $0.533 \pm 0.308$ & $0.601 \pm 0.293$ \\
Control & $0.511 \pm 0.281$ & $0.491 \pm 0.231$ & $0.470 \pm 0.249$ & $0.599 \pm 0.262$ & $0.617 \pm 0.276$ \\
\hline
\end{tabular}

Exp 1: Activities before education. Exp 2: Activities after education. 
Table 5. Comparison of within group variance and between group of PCA angle elbow of short service badminton in separate groups during test $(\mathrm{M} \pm \mathrm{SD})$

\begin{tabular}{lcccccc}
\hline \multicolumn{1}{c}{ Stages } & Variations & Sum squares & df & Mean Square & F & P-value \\
\hline Pretest & 1 & 0.207 & 2 & 0.104 & \multirow{2}{*}{1.372} & 0.266 \\
& 2 & 2.717 & 36 & 0.075 & & \\
Acquisition phase & 1 & 0.200 & 2 & 0.100 & 1.588 & 0.218 \\
Immediate retention phase & 2 & 2.268 & 36 & 0.063 & & \\
Remember phase after next 5 & 1 & 0.254 & 2 & 0.127 & 1.877 & 0.168 \\
days & 2 & 2.438 & 36 & 0.068 & & \\
Remember phase after next 9 & 2 & 0.049 & 2 & 0.025 & 0.305 & 0.739 \\
days & 1 & 2.898 & 36 & 0.081 & & \\
\hline
\end{tabular}

$\$$ The mean difference is significant at the 0.05 level. 1) Between group. 2) Within group.

According to the findings, the difference in the accuracy of the serve in the pre-test of both groups reflected the effect of aerobic exercise before training on the accuracy of the serve. Although a $35-\mathrm{mm}$ difference in the accuracy of the serve between the two groups was not statistically significant, it could show important differences. This difference was probably the result of aerobic exercise in Experimental Groupland showed an increase in the accuracy. In the pre-test, significant improvement was created in the aerobic exercise before and after training and without aerobic exercise (control group). This improvement could not be due to aerobic exercise because two experimental groups and particularly the control group did not do any exercise before acquisition. However, learning significantly occurred in the pre-test in such a way that errors were greatly reduced. According to the analysis of the steps of learning, the research samples in the pre-test were in the step of the cognitive learning. Therefore, significant improvements occurred in the pre-test. However, the research results were inconsistent with other findings in this regard. For example, Dietrich (2004) reported that aerobic exercise can be harmful for motor function (26). Some research considered these exercises useful for motor function (27) and some studies reported that these exercises had no effect (28).Also, Labban et al. (2011) reported that aerobic exercises before training can be more effective than aerobic exercises after training; our findings support this interpretation (12). Roig et al. (2012) achieved the opposite findings. They conducted a study and concluded that in the early stages of learning, there is no significant difference, but after seven days of participating in the retention tests, performing aerobic exercises after training has a greater effect on learning before the training (11).

By comparing the experimental groups with the control group, we conclude that the highest error in the test of acquisition and retention of the three groups is related to the errors in the five days after the intervention in Experimental Group 1, as well as immediate retention in Experimental Group 2 after 40 trials. Therefore, performing the intensity aerobic exercises before training at $65 \%$ to $70 \%$ of maximal oxygen consumption has a great refection performance and stabilizes memory. Memory consolidation for five days can create deep and durable learning and then motor memory loss occurs. It is not clear why these exercises have a significant impact on the acquisition and the immediate retention. In this case, aerobic exercise can improve the accuracy of the serve for up to nine days but the progress is very slow. Given that minor differences in the levels of performance make a considerable difference in the result of the race, this progress should be considered. The results of Experimental Group 2 also support this explanation. When the aerobic exercise is performed after training, the highest error rate occurs during 48 hours after the aerobic exercise. After this time, the little progress in five days of the aerobic exercise has still not reached the performance level of the acquisition, indicating the minimum memory loss in this period of time. 
The findings of the PCA-based coordination show the contribution of the elbow joints in the implementation of the movement pattern. Accordingly, there is no significant difference between the coordination for the movement range of the elbow joint in the two groups at any stage of the learning process, and these results confirm previous results. In fact, these findings indicate that the contribution of the movement range of the elbow for the coordinated pattern has not been changed despite the mean differences; therefore, aerobic exercise before and after low serve badminton training cannot affect the pattern for the movement range of the elbow joint. The coordinated movement is multi-dimensional and requires the movement of subsystems, the coordination of eye, hand, rocket, and ball (22), inter-segmental coordination (29), intra-limb coordination (30), and inter-limb coordination (31). Based on the limb-striking pattern, the shoulder, elbow, and wrist should have appropriate function to develop a proper pattern for coordinated movement. According to the findings of this study, aerobic exercise has a superficial effect on learning, only affects the output of movement behavior, and probably cannot create sustained effects at the pattern level for coordination in the joint or neural signals. In fact, there was no significant difference in the subjects at the measurement steps. The results of the pattern for coordination emphasize the sustainability of the pattern at different stages. It seems that, independently of the form of the training, players can repeat the serve skill in a similar pattern and the possible reason is the simplicity of the skill. There was no difference in the results of the precision test. Also, the adjustment in the coordination results of the elbow and precision results indicate the level of simplicity for coordination.

Roig et al. (2012) argued that no effect of aerobic exercise on the acquisition of motor skills in a short time may be related to fatigue caused by the aerobic exercise, leading to a reduction in the accuracy of the tracking task (11). Recent evidence suggests that moderate-intensity aerobic exercise may have harmful effects on the accuracy of performance (3).Some researchers have suggested that the choice of the type of cognitive training and protocol specification can be effective in improving performance (15).
Kantak et al. (2012) reviewed the findings of 41 studies on motor skills. The results showed that 19 studies indicate a significant difference between groups in the delayed retention tests (9).

One reason for the difference in findings is the type of task. The task for the short serve in badminton as a single task is different from the task used in the study of Roig et al. (2012). Roig et al. (2012) used the tracking and permanent task. The difference in the nature of the task in the present study and the task used in the study of Roig et al. (2012) may explain the differences in the findings (11). Roig et al. (2012) used the aerobic exercises at $65 \%$ to $70 \%$ of maximal oxygen consumption, similar to the present study. One of the findings was a significant difference in the memory consolidation test. No significant differences were observed in the acquisition and the immediate retention in the study conducted by Rouge et al. (2012). Their findings support the results of the present study. It seems that no significant impact of the aerobic exercise at $65 \%$ to $70 \%$ of maximal oxygen consumption on the acquisition and the immediate retention are due to the features of the task in the present study. It is likely that the lower-intensity aerobic exercise affects a single task, such as the short serve in badminton(11). For example, Joyce et al. (2009) used the aerobic exercise at $40 \%$ of maximal oxygen consumption but no significant results were observed after training (32).

There seems to be a kind of exchange between the effects of aerobic exercise on cognitive performance and the short serve. The research findings show the effect of aerobic exercise on immediate cognition $(33,34)$. They have proven this effect on motion performance in delayed retention (10). The results of the present study support these findings and indicate the effect of aerobic exercise on short serve performance (as a single motor activity) for up to fivedays. It can be concluded from these findings that the low cognitive load (such as performing the short serve in badminton) can reduce the impact of the aerobic exercise at $65 \%$ to $70 \%$ of maximal oxygen consumption on motor function. In contrast, increasing the cognitive load and time for performing tasks (such as the tracking task) enhances the effect of aerobic exercise on motor function. According to the obtained results, moderate-intensity aerobic exercise can have a 
positive effect on cognitive function, and on learning and memory processes $(15,35)$. Also, performing aerobic activity moderates memory and has a positive impact on it. The studies include the effect of aerobic exercise on shortterm memory $(10,36-38)$ and long-term memory $(12,37,39)$, which supports the hypothesis. The researchers argued that medium- and highintensity aerobic exercise can improve the speed of response in the working memory and has little or no harmful effect on the accuracy of movements. Decreasing accuracy may be due to an increase in the concentrations of catecholamine's, leading to an increase in motor noise and a decrease in accuracy (3). It is not clear if the cognitive-motor intervention in a simple task can be observed in other intensities of aerobic exercise by controlling the conditions of the experiment.

It should be noted that using the task of the serve in badminton as a single task can enhance the process of learning but cannot indicate the impact of the aerobic exercise well. One reason is the low impact of the aerobic exercise, which makes it difficult to measure. In fact, the maximum effect of the aerobic exercise is much less than the effect of the exercise on the samples. With regard to the content of the task in the study, the aerobic exercises are ineffective. In fact, this problem can be attributed to a lot of research in this area. Therefore, it is necessary to revise the methodology. This is a common challenge in studies on motor behavior (1). It is likely that the effect of aerobic exercises on accuracy can effectively be investigated using more limited tasks with lower degrees of freedom. According to these reasons, future research can use more limited tasks and those associated with lower learning to investigate the effects of aerobic exercise.

\section{CONCLUSION}

The results of the study indicated that aerobic exercises before training at $65 \%$ to $70 \%$ of maximal oxygen consumption is more effective than aerobic exercises after training in reducing the rate of motion error, increasing accuracy, and improving the short serve in badminton. It is likely that this result is true for other short-term tasks. An idea can be used to compare the single and continuous tasks in terms of the effectiveness of aerobic exercise at $65 \%$ to $70 \%$ of maximal oxygen consumption and to control these effects. The findings could also potentially be affected by a cognitive-motor intervention. The type of the task and the cognitive requirements can determine the level of the intervention. However, more research is needed to investigate the exact effects of different intensities of aerobic exercises on various tasks in terms of the features of the motor control and provide practical results.

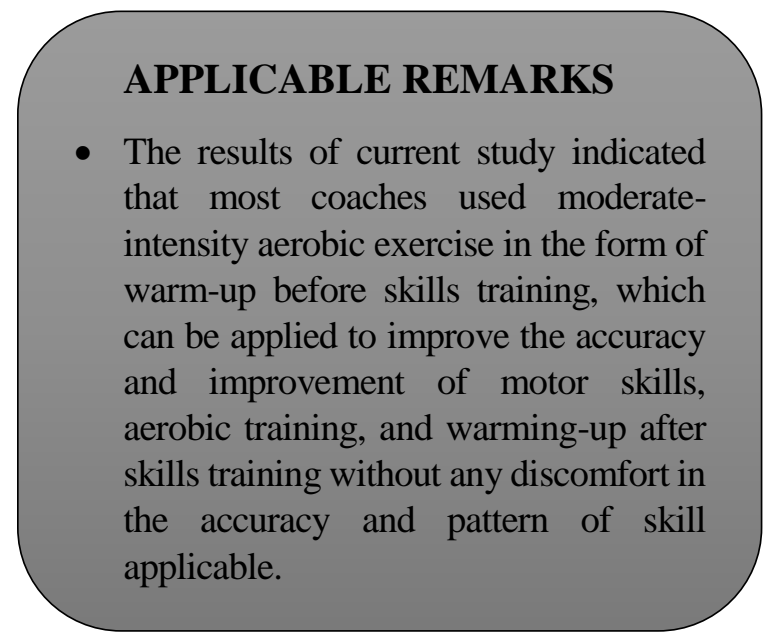

\section{REFERENCES}

1. Schmidt RA, Lee T. Motor control and learning: Human kinetics; 1988.

2. Brisswalter J, Collardeau M, René A. Effects of acute physical exercise characteristics on cognitive performance. Sports Medicine. 2002;32(9):555-66.

3. McMorris T, Sproule J, Turner A, Hale BJ. Acute, intermediate intensity exercise, and speed and accuracy in working memory tasks: a meta-analytical comparison of effects. Physiology \& Behavior. 2011;102(3):421-8.

4. Lucas SJ, Ainslie PN, Murrell CJ, Thomas KN, Franz EA, Cotter JD. Effect of age on exercise-induced alterations in cognitive executive function: relationship to cerebral perfusion. Experimental Gerontology. 2012;47(8):541-51.

5. Tomporowski PD. Effects of acute bouts of exercise on cognition. Acta Psychologica. 2003;112(3):297-324.

6. Cotman CW, Berchtold NC, Christie L-A. Exercise builds brain health: key roles of growth factor cascades and inflammation. Trends in Neurosciences. 2007;30(9):464-72.

7. Walker EL. Action decrement and its relation to learning. Psychological Review. 1958;65(3):129. 
8. Cian C, Barraud P, Melin B, Raphel C. Effects of fluid ingestion on cognitive function after heat stress or exerciseinduced dehydration. International Journal of Psychophysiology. 2001;42(3):243-51.

9. Kantak SS, Winstein CJ. Learning-performance distinction and memory processes for motor skills: A focused review and perspective. Behavioural Brain Research. 2012;228(1):219-31.

10. Tomporowski PD, Cureton K, Armstrong LE, Kane GM, Sparling PB, Millard-Stafford M. Short-term effects of aerobic exercise on executive processes and emotional reactivity. International Journal of Sport and Exercise Psychology. 2005;3(2):131-46.

11. Roig M, Skriver K, Lundbye-Jensen J, Kiens B, Nielsen JB. A single bout of exercise improves motor memory. PlosOne. 2012;7(9):e44594.

12. Labban JD, Etnier JL. Effects of acute exercise on long-term memory. Research Quarterly for Exercise and Sport. 2011;82(4):712-21.

13. Hillman CH, Pontifex MB, Raine LB, Castelli DM, Hall EE, Kramer AF. The effect of acute treadmill walking on cognitive control and academic achievement in preadolescent children. Neuroscience. 2009;159(3):1044-54.

14. Kamijo K, Hayashi Y, Sakai T, Yahiro T, Tanaka K, Nishihira Y. Acute effects of aerobic exercise on cognitive function in older adults. The Journals of Gerontology Series B: Psychological Sciences and Social Sciences. 2009;64(3):356-63.

15. Lambourne K, Tomporowski P. The effect of exercise-induced arousal on cognitive task performance: a metaregression analysis. Brain Research. 2010;1341:12-24.

16. Button C, Davids K, Schollhorn W. Coordination profiling of movement systems. 2006.

17. Morasso P. Spatial control of arm movements. Experimental Brain Research. 1981;42(2):223-7.

18. Müller H, Sternad D. Motor learning: changes in the structure of variability in a redundant task. Progress in Motor Control: Springer; 2009. p. 439-56.

19. Wilson BN, Crawford SG, Green D, Roberts G, Aylott A, Kaplan BJ. Psychometric properties of the revised developmental coordination disorder questionnaire. Physical \& Occupational Therapy in Pediatrics. 2009;29(2):182-202.

20. Panahi Broujeni E, Shojaei M, Daneshfar A. Effects of Feedback after Successful Trials, Normative Feedback, and Self-Controlled Feedback on Learning of Badminton Service. International Journal of Sport Studies. 2014;4(7):823-9.

21. Winter DA. Biomechanics and motor control of human movement: John Wiley \& Sons; 2009.

22. Johansson RS, Westling G, Bäckström A, Flanagan JR. Eye-hand coordination in object manipulation. Journal of Neuroscience. 2001;21(17):6917-32.

23. Winter B, Breitenstein C, Mooren FC, Voelker K, Fobker M, Lechtermann A, et al. High impact running improves learning. Neurobiology of Learning and Memory. 2007;87(4):597-609.

24. Sjöberg H. Physical fitness and mental performance during and after work. Ergonomics. 1980;23(10):977-85.

25. Tomporowski PD, Ellis NR, Stephens R. The immediate effects of strenuous exercise on free-recall memory. Ergonomics. 1987;30(1):121-9.

26. Dietrich A, Sparling PB. Endurance exercise selectively impairs prefrontal-dependent cognition. Brain and Cognition. 2004;55(3):516-24.

27. Pesce C, Capranica L, Tessitore A, Figura F. Focusing of visual attention under submaximal physical load. International Journal of Sport and Exercise Psychology. 2003;1(3):275-92.

28. Tomporowski PD, Davis CL, Lambourne K, Gregoski M, Tkacz J. Task switching in overweight children: effects of acute exercise and age. Journal of Sport and Exercise Psychology. 2008;30(5):497-511.

29. Borghese NA, Bianchi L, Lacquaniti F. Kinematic determinants of human locomotion. The Journal of Physiology. 1996;494(Pt 3):863-79.

30. Cirstea M, Mitnitski A, Feldman A, Levin M. Interjoint coordination dynamics during reaching in stroke. Experimental Brain Research. 2003;151(3):289-300.

31. Ivry R, Diedrichsen J, Spencer R, Hazeltine E, Semjen A. A cognitive neuroscience perspective on bimanual coordination and interference. Neuro-behavioral determinants of interlimb coordination: Springer; 2004. p. 25995.

32. Joyce J, Graydon J, McMorris T, Davranche K. The time course effect of moderate intensity exercise on response execution and response inhibition. Brain and Cognition. 2009;71(1):14-9.

33. Hogervorst E, Riedel W, Jeukendrup A, Jolles J. Cognitive performance after strenuous physical exercise. Perceptual and Motor Skills. 1996;83(2):479-88.

34. Lichtman S, Poser EG. The effects of exercise on mood and cognitive functioning. Journal of Psychosomatic Research. 1983;27(1):43-52. 
35. Roig M, Nordbrandt S, Geertsen SS, Nielsen JB. The effects of cardiovascular exercise on human memory: a review with meta-analysis. Neuroscience \& Biobehavioral Reviews. 2013;37(8):1645-66.

36. Pontifex M, Hillman C, Fernhall B, Thompson K, Valentini T. The effect of acute aerobic and resistance exercise on working memory. Medicine and Science in Sports and Exercise. 2009;41(4):927.

37. Coles K, Tomporowski PD. Effects of acute exercise on executive processing, short-term and long-term memory. Journal of Sports Sciences. 2008;26(3):333-44.

38. Sibley BA, Beilock SL. Exercise and working memory: an individual differences investigation. Journal of Sport and Exercise Psychology. 2007;29(6):783-91.

39. Potter D, Keeling D. Effects of moderate exercise and circadian rhythms on human memory. Journal of Sport and Exercise Psychology. 2005;27(1):117-25.

Darzabi, T., Taheri, H. R., Saberi Kakhki, A. R. (2018). Ann Appl Sport Sci, 6(1): 37-46. 